\title{
A Preference Elicitation Method Based on Bipartite Graphical Correlation and Implicit Trust
}

\author{
Quanli $\mathrm{Gao}^{\mathrm{a}}$, Ling Gao ${ }^{\mathrm{a}, *}$, Jianping Fan ${ }^{\mathrm{a}, \mathrm{b}}$, Jie Ren $^{\mathrm{a}}$ \\ ${ }^{a}$ Service computing and Application lab,Department of information science and technology, Northwest \\ University, 710127,PR China \\ ${ }^{b}$ Department of Computer Science, The University of North Carolina at Charlotte, NC28223, United States
}

\begin{abstract}
In the age of big data, information overload is getting worse. Most of the existing recommender systems which apply data analysis and behavioral analysis to make personal recommendation have suffered from the problem of low prediction accuracy. To address this problem, a new preference elicitation algorithm is developed by using bipartite graphical correlation and implicit trust. More precisely, to compute the bipartite graphical correlation, an improved single-source shortest path is firstly presented to get the shortest behavior path based on the user-item bipartite graph. While the Markov separation algorithm is employed to separate uncorrelated vertexes and obtain the values of bipartite graphical correlation. Secondly, the implicit trust is used to represent trust relationship between users and items which have no historical behaviors. Independent trust group is applied to represent the closed-loop path on the user-item bipartite graph, and a compute method is developed to get the values of implicit trust based on the trusty values of independent trust group and explicit trust. Finally, a weighted prediction method based on bipartite graphical correlation and implicit trust is employed to compute the final ratings. Our empirical experiments are performed on a sparse data set, the results of which have demonstrated that our method can achieve lower $P @ R$ and efficiently improve recommendation quality.

Keywords: Recommender system, Independent trust group, Explicit trust, Implicit trust, Bipartite graphical correlation
\end{abstract}

\footnotetext{
${ }^{*}$ Corresponding author at:Service computing and Application lab,Department of information science and technology, Northwest University,710127,PR China

Email address: gl@nwu.edu.cn (Ling Gao)
} 


\section{Introduction}

The amounts of information resources are increasing far more quickly than our ability to process them. It is very important to develop new technologies to make sure that users can get their preferential resources. The best way to address this problem is to use recommender system which is becoming a very important and necessary part of digital resource providers. Their main goal is to help users to find personalized preference through suitable recommendation algorithms. Over the past decades, various approaches have been developed to support personalized recommendation. Generally, they can be categorized into two classes: (1) Content-based recommendation algorithms[1, 2, 3], which get predictions by using mathematical models and data mining to model users' preference based on their historical behavior records. (2) Collaborative filtering $[4,5]$, which filters the uncorrelated items based on a collection of similar users' rating history to generate prediction. Those approaches make great progress of recommendation technologies, but they still encounter many drawbacks, such as the singleness of users' interest in content-based approaches, the sparsity problem and cold-start problem in collaborative filtering based recommender systems $[6,7]$.

Cold-start problem can be separated into two categories, cold-start item and cold-start user. Taking the first kind as an example, it describes new items in digital information systems which contain very few users' historical behavior records. In this situation, recommender systems are generally unable to recommend them to suitable users. The sparsity of data set is another well-known drawback for the collaborative filtering based recommender systems, because most users have not rated enough items, hence the user-item historical preference records are very sparse, and the information that recommender systems can use is inadequate. This drawback leads directly to the powerless to distinguish users' preference. To tackle these problems, many approaches have been proposed in recent years. For examples, Wiranto et al.[8] presented a multidimensional collaborative filtering method to improve the way that measures the similarities among users. Anand et al.[9] used graph-based global similarity estimates to improve the accuracy of recommendation. Luo et al.[10]. proposed a non-negative matrix factorization based collaborative filtering method. Kim et al.[11] utilized collaborative tagging to solve the sparsity problem. Leung et al.[12] applied sentiment analysis to augment ratings to perform the collaborative filtering. Wei et al.[13] adopted K-means clustering to cluster items into different classes and introduce the factor of overlap to optimize the accuracy of the local similarities among users. Wang et al.[14] presented a machine learned ranking function to generate recommendation on 
micro-blogging systems. Zhao et al.[15] proposed a ranking algorithmic framework for heterogeneous manifold in social tagging services. Kim et al.[16] used rating normalization to improve collaborative filtering based recommender systems. In these methods mentioned above, users' historical preference is often described as user-item rating matrix, and the prediction is generated by process and analysis of the matrix. However, the influence relation among users' preference will become very complex and hard to compute with the increasing number of users and items. Inspired by graph-based model, many researchers use the user-item bipartite graph to describe the historical preference[17, 18, 19], representing the standard user-item rating matrix as graph vertexes and edges to get predictions. Such as, Liu et al.[17] focus on the combination of collaborative filtering and network structure, Lee et al.[18] try to find novel recommendations among relevant items based on graph model, Zhong et al.[19] study on the directed trust graph based recommendation methods. Their experimental results proved that graph-based algorithms are excellent in mining the complex relationship among users.

In this paper, we address those issues by merging the correlation level and trust relationship which are described as the relationship of user-item and user-user respectively. The correlation level between users and items is similar to the same relationship in item-based collaborative filtering, which describes whether users are fond of items, since there is a high probability that users are favor of items which relate to their historical rated items. We compute the correlation level through the Markov separate algorithm which is based on the user-item bipartite graph and improved Dijkstra algorithm. The trust relationship among users describes the similarity of users' preference which is reflected by the historical preference to the same items from different users. We classify the trust relationship into explicit trust and implicit trust based on whether the users have historical behavior records on same items. Then it merges the correlation level and trust level by introducing a weighted coefficient to get the final predictions. Several experiments based on the sparse data set demonstrate that the proposed method can achieve lower $P @ R$, and efficiently improve recommendation quality. The contributions of this paper can be summarized as follows:

(1) The most relevant items for users are extracted by the approach of bipartite graphical correlation based on the user-item bipartite graph.

(2) A measure method of trust relationship is provided by using the explicit trust, implicit trust and independent trust group.

(3) The measure method and influence factor of independent trust group are proposed for the first time. 
The remainder of this paper is organized as follows: The second part of this paper describes the proposed method and provides a detailed description of how the approach uses those factors to get the bipartite graphical correlation, explicit trust and implicit trust. In the third section, we present the effectiveness of our algorithm compared with existing works. The final section provides conclusion, discussion and direction for our future work.

\section{Bipartite graphical correlation and implicit trust based recommender system}

The motivation of using graph model and trust model to generate prediction can be divided into two categories. The first is to mine the complex relationship among users-users, and usersitems deeper, which emphasizes the influence of users' historical behaviors on the interaction among users-users and users-items, and shifts focus from the interaction relationship to the recommender algorithms themselves. The second motivation is to overcome the limitations and drawbacks suffered by most of the existing recommender systems. In which the algorithms adopted only rely on the extremely sparse user-item rating data, and the computed similarity in collaborative filtering based recommender systems will be potentially unreliable and incorrect $[16$, 20]. For example, new users can not get their favorite items until the systems have acquired the preference from their historical behavior records [21], and new items cannot be recommended to the suitable users until enough users have behaviors (browse, download, give a mark) on them.

\subsection{The User-item bipartite graph}

The purposes of recommender systems are to predict the values of certain items and to recommend new items for particular users according to their previous preference and other users who share similar options. Users' historical preference, which has played a very important role in obtaining preference processes, is almost the only data source for recommender systems. In a typical collaborative filtering based scenario[22, 23], there is a list of users $U=\left\{U_{1}, U_{2}, \cdots, U_{n}\right\}$, and a list of items $I=\left\{I_{1}, I_{2}, \cdots, I_{m}\right\}$, in which every users' historical preference on different items is saved in the user-item matrix as certain numerical rating. But in a graph-based scenario, users' historical preference is represented by series of user-item tuples which are random distribution. Assume that the set of users' historical preference is $U I=\left\{\left(V_{u}, V_{i}\right) \mid V_{u} \in U, V_{i} \in I\right\}$, in which $U$ and $I$ represent the users set and items set respectively. For example, tuple $\left(V_{u}, V_{i}\right)$ represent user $u$ 's historical preference on item $i . G B(V, E)$ is the user-item bipartite graph of $U I$, where $V=\left\{V_{u} \cup V_{i} \mid V_{u} \subseteq U, V_{i} \subseteq I\right\}$, and $E=\left\{e\left(V_{u}, V_{i}\right) \mid V_{u} \in U, V_{i} \in I\right\}$ is the set of 
edges in $G B$. For every $\left(V_{u}, V_{i}\right) \in U I$ there is an edge $e\left(V_{u}, V_{i}\right) \in E$ in $G B$ corresponding to it. The graphical expression is depicted in Fig.1.

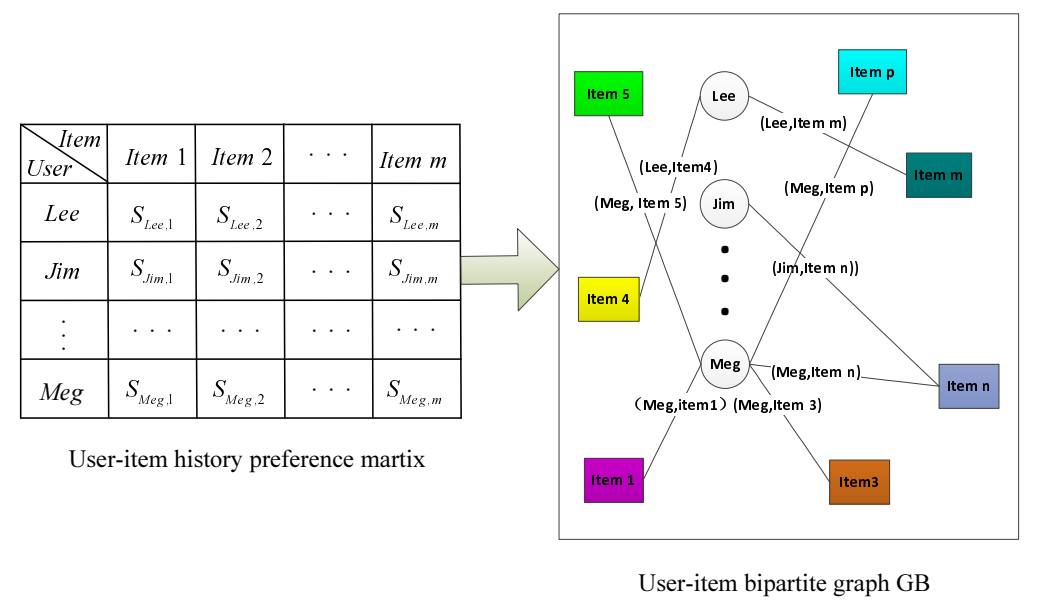

Figure 1: An example of the transformation from user-item preference matrix to user-item bipartite graph

As one traditional prediction method, collaborative filtering firstly computes the similarities between users or items. Taking users' similarities as an example, the main idea of similarity computation is to extract the users who have rated same items by using a similarity computation algorithm, such as cosine-based similarity, correlation-based similarity, and adjusted cosines similarity. And then it gets predictions based on the historical choices of the most similar users $[15,17,20]$. Based on the user-item bipartite graph, this processing is transformed to compute the values of correlation between user $u$ and items which have no historical preference by user $u$ on the user-item bipartite graph. The bigger the values of correlation are, the larger the probability that this item can be recommended to user $u$ is. For example, to any user $u, p$ and $q$ are items which have no historical preference records of user $u$. If there are more ways that connect item $p$ to user $u$ than item $q$ to user $u$, which means that item $p$ is more correlative than $q$, then item $p$ is prior to $q$ in the recommendation list of user $u$.

The main factors that influence the values of correlation among users (items) can be divided into three parts. Take user $m$ and user $n$ as an example

(1) The amount of paths between user $m$ and user $n$.

(2) The length of weighted edges between user $m$ and user $n$.

(3) The amount of vertexes on the shortest path between user $m$ and user $n$.

The larger the amount of paths among users is, the larger the correlation is. The paths among user vertexes mean the intersection of users' historical preference. The larger amount 


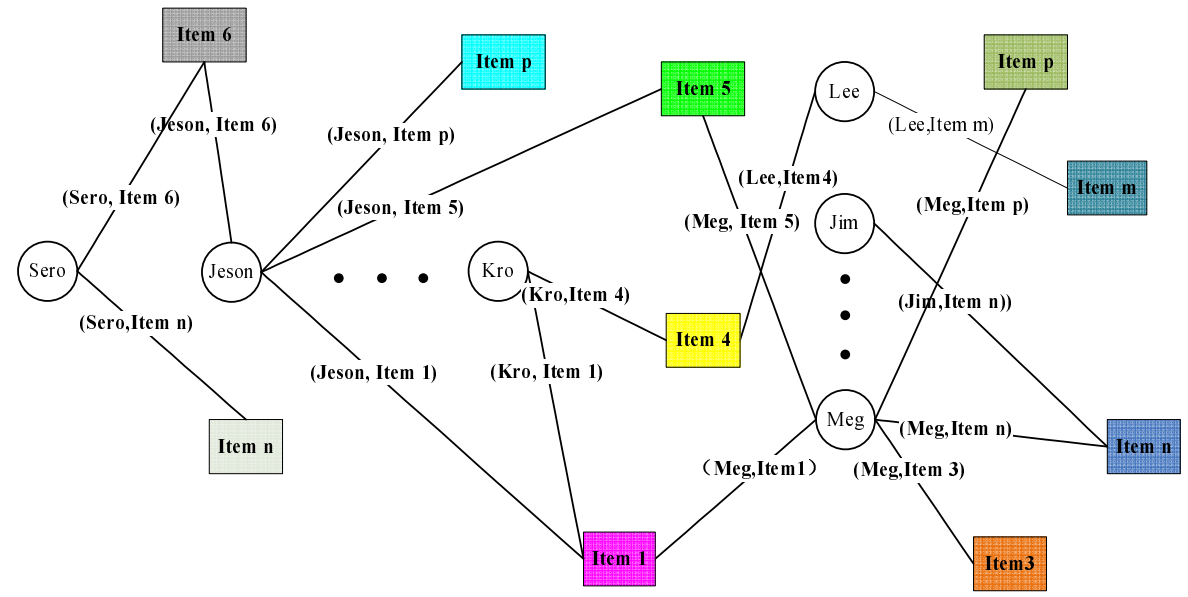

Figure 2: An example of correlation among vertexes

of it indicates that we have adequate preference records to measure their credible correlation, especially under sparsity data. As shown in Fig.2, we take users Kro, Jeson, Sero as an example. The amount of paths between Kro and Jeson is two. They are Kro $\rightarrow$ Item $1 \rightarrow$ Jeson, Kro $\rightarrow$ Item $1 \rightarrow \mathrm{Meg} \rightarrow$ Item $5 \rightarrow$ Jeson respectively. This amount between Kro and Sero is one. It is Kro $\rightarrow$ Item $1 \rightarrow$ Jeson $\rightarrow$ Item $6 \rightarrow$ Sero. The values of correlation between Kro and Jeson are more than that between Kro and Sero, which means Jeson may has much bigger influence on the preference of Kro than Sero. The length of weighted edger among users is another factor that influence the correlation[24]. As shown in Fig.2, if (Jeson,Item 1) is bigger than (Meg,Item 1), item 1 will be recommended to users who correlate to Jeson instead of users who correlate to Meg. The bigger the amount of vertexes on the shortest path among users is, the more similar among the users preference is. This amount of vertexes between Kro and Jeson is one, while the amount between Kro and Sero is three, which indicates that items that meet the preference of Jeson have bigger probability than Sero that meat the preference of Kro. Judging from what have been argued above, the common characters of vertexes with higher correlation can be summarized as follows:

(1) There are many paths between each two vertexes.

(2) The shorter length of the shortest path between each two vertexes.

(3) The bigger values between each tuple.

\subsection{Computing the bipartite graphical correlation}

The idea of using bipartite graphical correlation to describe the correlation between users and items is inspired by the Markov-chain model. In the chain graph, the global Markov is often 
employed to separate different vertex sets. We carry out a bipartite graph based method to get the correlation values between users and items. To be specific, firstly, this method obtains the shortest behavior path between users and items which have no preference record by the users. Then it computes the values of bipartite graphical correlation through the improved Markov separate algorithm. The definitions that used in the method are defined as follows.

Definition 1. The in-degree and out-degree in the user-item bipartite graph. The definition of in-degree in graph theory is the number of vertexes which terminate at the vertex. The outdegree is the number of edges which start with the vertex. But in the user-item bipartite graph, only item vertexes have in-degree, and user vertexes only have out-degree. The new definition of in-degree $I d_{i}$ is the number of users who have historical behaviors on item $i$, and out-degree $O d_{u}$ describes the number of items which has user $u$ 's historical behavior record.

Definition 2. The shortest behavior path. It describes the shortest path between two vertexes. If we let user $u$ be the starting point, item $i$ be the ending point, and the single-pass behavior record be the least measuring unit which is also the edge with only two vertexes, then the shortest behavior path between $u$ and $i$ can be defined as the path which has the character of optimal substructure. The definition is as follows:

If $S_{u i}$ is the shortest path between user $u$ and item $i, m$ and $n$ are any two vertexes belonging to $S_{u i}, S_{m n}$ be the shortest path between $m$ and $n$. Then $S_{u i}$ has the character of optimal substructure between user $u$ and item $i$. It can be proved on the user-item bipartite graph as follows:

If $S_{u i}=\left\{V_{u}, \cdots, V_{k}, V_{s}, \cdots, V_{i}\right\}$ is the shortest path between $V_{u}$ and $V_{i}$, assume $V_{k}$ and $V_{s}$ are two vertexes on this path, then there must be $S_{u i}=S_{u k}+S_{k s}+S_{s i}$. If $S_{k s}$ is not the shortest path between $V_{k}$ and $V_{s}$, there will be another shortest path $S_{k s}^{\prime}$ between $V_{k}$ and $V_{s}$, then $S_{u i}^{\prime}=S_{u k}+S_{k s}^{\prime}+S_{s i}<S_{u i}$, which is contradictory to the prior assumption that $S_{v i}$ is the shortest path between $V_{u}$ and $V_{i}$.

To compute the shortest behavior path, we need to find the optimal substructure between user $u$ and item $i$. In this paper, we use the improved single-source shortest Path, Ibu-Dijkstra, to get it. Basing on the users' historical preference $(u, j),(u, j) \in U I$, this method generates the vertexes which belong to the optimal substructure one by one. The detailed process is as follows:

To any starting vertex $V_{u}$, we choose the vertex $V_{m}$, since it is the directly adjacent vertex to $V_{u}$, and also has the least edge value(in-degree or out-degree). Then the shortest behavior path between $V_{u}$ and $V_{i}$ can be defined as below:

$$
S_{u i}=\min \left\{S_{u i}, S_{u m}+\text { matrix }\left[V_{i}\right]\left[V_{m}\right]\right\}
$$


where $S_{u i}$ represents the shortest behavior path between $V_{u}$ and $V_{i} . S_{u m}$ is the same path between $V_{u}$ and $V_{m}$.

(1) Select the vertex $V_{m}$ from vertex set $V$ which has the least value of $S$ compared with $V_{u}, S$ is the shortest behavior path between any vertexes $V_{u}$ and $V_{m}$.

(2) Update the vertexes' values of $S$ which are directly next to $V_{m}$.

(3) Repeat (1) and (2) until it traverses the vertex set $V$ completely.

Definition 3. Markov separation. Any of user-item bipartite graph $G B$ can be translated into a graphical independence model by a given separation criterion $S C$, as follows:

$(X, Y \mid H) \in M_{S C}(G B) \Leftrightarrow$ The vertex sets $X$ and $Y$ of $G B$ are separated by $S C$

The separation criterion $S C$ is the global Markov of $G B$ in graph theory, which means that to any vertex set $P$ and $Q(P, Q \subset V)$ sharing no common vertexes. We assume that all the possible paths between $P$ and $Q$ share common vertexes, and these common vertexes constitute the vertex set $B(B \subset V)$. In other words, to any vertexes $V_{m}$ and $V_{n}\left(V_{m} \in P, V_{n} \in Q\right)$, every path between $V_{m}$ and $V_{n}$ has non-empty intersection with $B$ (in this situation $B$ is the separation criterion of $S C$ ), which indicates that vertexes $P$ and $Q$ are separated by separation criterion $B$. Basing on this, we propose a separation criterion $S C_{G B}$ for user-item bipartite graph $G B$ to separate uncorrelated vertexes including item vertexes and user vertexes, and obtain the values of bipartite graphical correlation.

To any user vertex $V_{u}$, we assume that $P a t h_{u}$ is the set of shortest behavior path between $V_{u}$ and other vertexes, such as $V_{i}$ got from Ibu-Dijkstra, then the bipartite graphical correlation between $V_{u}$ and $V_{i}$ is measured by:

$$
Q_{u i}= \begin{cases}\frac{P_{u i}}{\left|\left(V_{u i}-\alpha\right)\right|+\beta \cdot B V_{u i}} & \text { At least one path between } V_{u} \text { and } V_{i} \\ 0 & \text { Else }\end{cases}
$$

where $P_{u i}$ is the number of paths between $V_{u}$ and $V_{i} . V_{u i}$ represents the number of vertexes on Path $_{u} . \alpha$ and $\beta$ are corrected parameters. $B V_{u i}$ is the sum of behavior values, which can be denoted as:

$$
B V_{u i}=\sum_{j \in V_{u i}} S_{u j}
$$

where $V_{u i}$ is the vertex set on $P a t h_{u} . S_{u j}$ is often described as rating scoring, represents the historical preference between user $V_{u}$ and item $V_{j}$. The exclusion rule(separation criterion $S C_{G B}$ ) of item vertexes can be defined as: 
(1) Choose the top $n$ vertexes based on the values of $Q_{u i}$ and exclude other vertexes.

(2) Hot items means the items having much more users' historical preference records than other items. For hot items, there is a bigger probability that users have historical behavior on them in other web sites or applications. To reduce the Matthew Effect, we introduce the penalty strategy to avoid the influence of hot items which have larger in-degree. The penalty strategy is given as follows:

$$
Q_{u i}= \begin{cases}Q_{u i} \cdot\left(\frac{I d_{t h d}}{I d_{i}+I d_{t h d}}\right)^{2} & \text { If } I d_{i} \geq I d_{t h d} \\ 0 & \text { Else }\end{cases}
$$

where $I d_{i}$ is the in-degree of $V_{i} . I d_{t h d}$ is the penalty threshold value, which changes with the different application environments.

\subsection{Compute the implicit trust}

In the field of recommender system, the definition of trust is different from other fields, such as information security. If user $V_{m}$ considers that there is a bigger possibility to obtain the items which fit with his/her preference according to user $V_{n}$ 's historical preference, then $V_{n}$ is trusty for $V_{m}$. The underlying assumption of trust is that users' historical behaviors can represent how they will act in the future. In this paper, we measure the trust between users and items by individual reliability and group reliability which are got from the correlation of historical preference. In the graph $G B$, the correlations among historical preference are represented by the values of edges among vertexes which are often described by the rating values. In this section, the method classifies the trust as explicit trust and implicit trust based on the non-binary trust graph, and then the independent trust group based on the closed-loop path of users' historical preference is applied to compute the explicit trust and implicit trust. The graphical description is shown in Fig.3. Some concepts in the model are defined as follows:

Definition 4. Non-binary trust graph. It describes the improved user-item bipartite graph $G B$ whose values of edges are represented by the users' historical preference, and the values are not limited to 0 and 1. As shown in Fig.4, it is same to the $G B$ on patterning.

Definition 5. Explicit trust and implicit trust. The explicit trust describes the relationship between user $V_{u}$ and item $V_{i}$ (taken as an example). If $V_{u}$ has historical behavior on $V_{i}$, such as Jeson $\rightarrow$ Item p, Kro $\rightarrow$ Item 5, and Jim $\rightarrow$ Item 1 in Fig.4, the values of explicit trust can be measured by the historical preference. On the contrary, the implicit trust represents that there is no historical behavior between $V_{u}$ and $V_{i}$. If you want to connect $V_{u}$ and $V_{i}$, you need to go 


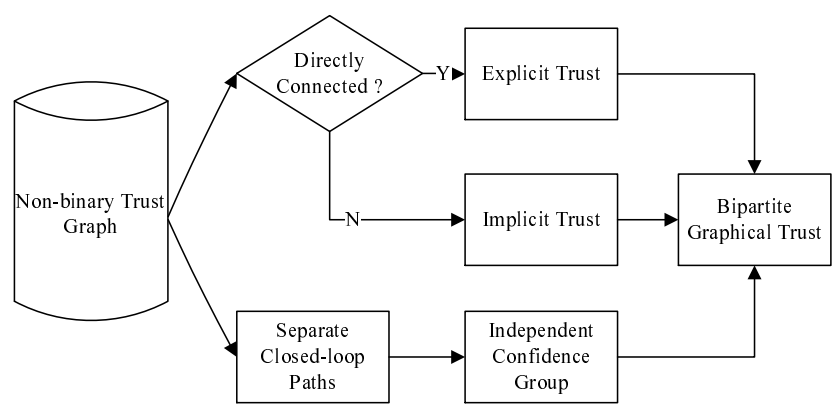

Figure 3: The processing of compute the implicit trust

through at least two edges in $G B$, such as Jeson $\rightarrow$ Item 1 , Sero $\rightarrow$ Item n, and Meg $\rightarrow$ Item $\mathrm{p}$ in Fig.4.

The explicit trust describes the trust relationship among vertexes which are directly adjacent. Take user $V_{u}$ and item $V_{i}$ as an example, in order to avoid the influence of different users' rating scales and items' inherent attributes, we propose a computing method to get the values of explicit trust as follows:

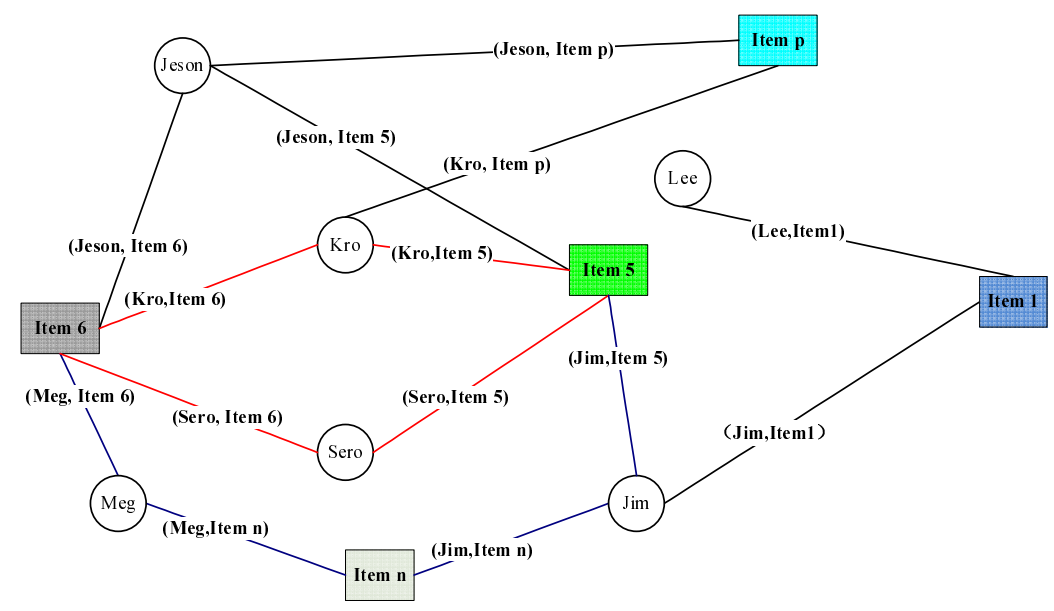

Figure 4: Example of the trust relationship between users and items

$$
E T_{u i}=1-\frac{\overline{S_{u}}+\overline{S_{i}}}{\sqrt{\left(S_{u i}-\overline{S_{i}}\right)^{2}+\left(S_{u i}-\overline{S_{u}}\right)^{2}}}
$$

where $S_{u i}$ represents user $V_{u}$ 's preference value on item $V_{i}, \overline{S_{u}}$ is the average historical preference value on other items, $\overline{S_{i}}$ describes users' average historical preference value on item $V_{i}$. 
The implicit trust describes the trust relationship among vertexes which are not directly adjacent. Those vertexes are other important influence factors which represent users' preference. Take user $V_{u}$ and item $V_{i}$ for example, we analyze all the vertexes which correlate to $V_{u}$ and $V_{i}$, and can be separated from different closed-loops, in which each closed-loop has its own trust values. In this paper, we use the number and the trust values of independent trust groups on the shortest behavior path between users and items which have no direct edges to get the values of implicit values. Some involved concepts are defined as follows:

Definition 6. Independent trust group. It describes the closed-loop path on the useritem bipartite graph, such as Kro $\rightarrow$ Item $5 \rightarrow$ Sero $\rightarrow$ Item $6 \rightarrow$ Kro, and Jeson $\rightarrow$ Item $6 \rightarrow$ Kro $\rightarrow$ Item $\mathrm{p} \rightarrow$ Jeson in Fig.4. Every independent trust group has it's own group credibility which is affected by the number of vertexes and values of edges on the loop. The larger the number of vertexes in the loop is, the lower the group credibility is. Because if there are too many vertexes in the group, there may be a long path between any two vertexes which are not directly adjacent, the correlation and the similarity of preference will be reduced. On the other hand, the larger the values of edges are, the bigger the group credibility is, because the values of edges represent users' historical preference.

To any group $M$, the group credibility can be denoted as:

$$
G T_{M}= \begin{cases}\sum_{i \in M} G V_{u i} & \\ \frac{V N_{M}-3}{} & \text { If exist closed-loop } \\ 0 & \text { Else }\end{cases}
$$

where $V_{u}$ is a starting point in $M . V_{i}$ describes the directly adjacency vertex of $V_{u} . V N_{M}$ represents the number of vertexes in group $M . G V_{u i}$ is the value of edge $\left(V_{u}, V_{i}\right)$ in the independent trust group. The value of $G V_{u i}$ is influenced by the explicit trust and the number of independent trust groups which include the edge $\left(V_{u}, V_{i}\right)$. It can be denoted as follows:

$$
G V_{u i}=\frac{C_{u i}}{C_{t h d}+C_{u i}} \cdot E T_{u i}
$$

where $C_{u i}$ is the number of independent trust groups which include edge $\left(V_{u}, V_{i}\right) . C_{t h d}$ is the corrected parameter.

Then the calculation of user $V_{u}$ 's implicit trust on item $V_{i}$ can be described as:

$$
I T_{u i}=\sum_{M \in M_{u i}} G T_{M}
$$


where $M_{u i}$ is the set of independent trust group which include the edge $\left(V_{u}, V_{i}\right)$.

\subsection{Get final preference}

In this section, we combine the bipartite graphical correlation $Q_{u i}$ and implicit trust $I T_{u i}$ to get users' preference. The graph correlation generates items which fit with users' preference by excluding the irrelevant items from the view of graph model, and the implicit trust measures the most valuable items for users from the view of trust relationship. Both of them are try to figure out the most suitable items for users. And both of them have positive impact on users' preference, which means that the bigger the $Q_{u i}$ and $I T_{u i}$ are, the larger probability that item $V_{i}$ meet user $V_{u}$ 's interest. The final preference computation can be denoted as:

$$
R_{u i}=Q_{u i}+\chi \cdot I T_{u i}
$$

where $\chi$ represents the weighting parameter, whose value can be automatically adjusted by the system(in this paper), or it can be given by users. We recommend Top- $n$ items to user $V_{u}$ who has the largest $R_{u i}$.

\section{Experimental results}

In this section, a series of experiments are conducted to evaluate algorithms proposed in this paper. We verify the following questions by employing those experiments.

(1) The best corrected parameters to compute the bipartite graphical correlation.

(2) The optimal weight of bipartite graphical correlation and implicit trust to gets users' preference.

(3) Whether the proposed algorithm can achieve better recommendation accuracy.

\subsection{Data set}

Our experiments employ the real-world data set MovieLens, which includes about 943 users' historical rating records on 1682 movies, and the sparsity of this data set is 100000/(9431682) = 0.06. MovieLens uses ratings (1-5) to represent the users' preference for the movies, which means that the larger ratings the user gives, the more the user likes the movie. Each user has rated at least 20 movies. To solve the unconvincing experimental result caused by the single data set, we use the ALL BUT ONE(ABO) algorithm to conceal the users' historical preference data randomly. When the proposed algorithm uses the data set, ABO will conceal some records of 
each user randomly. Besides, to test the applicability of our algorithm, we separate the data set into training set and test set with different scales, in which the first set is used to train the parameters of model, and the last one is used to test the model. Hence, there are many experimental results. After repeating experiments and tests, we select the representative results, in which $40 \%$ and $60 \%$ of this data set are selected as training sets while others as the test sets respectively to verify our algorithm.

\subsection{Evaluation metrics}

Recommender systems have different measurements to evaluate the quality of recommendation. In this paper, we employ the $P @ R$ to measure our prediction. As a representative example of statistical accuracy criterion, $P @ R$ measures the accuracy by contrasting the $R$ items that the user used most frequently with other $R$ items which are recommended by our algorithm. Then $P @ R$ can be defined by

$$
P @ R=\frac{\text { the number of items in predicted set which included in actual set }}{R}
$$

\subsection{Experiment results and analysis}

Experiment 1. Impact of independent trust group

In this section, the experiment is designed to test whether users on the independent trust group have bigger similarity of preference behavior, to test whether the independent trust group have influence on users' preference, and to verify the assumption of our method. We employ cosine similarity[8] to measure the similarities among users. Take user $U_{a}$ and $U_{b}$ as an example, if $I_{a b}$ denotes the common item set in which $U_{a}$ and $U_{b}$ both have historical preference records, the cosine similarity will use the average preference deviation on $I_{a b}$ to measure the preference similarity. It is defined as follows:

$$
\operatorname{Sim}(a, b)=\frac{\sum_{i \in I_{a b}} S_{a i} \cdot S_{b i}}{\sqrt{\sum_{i \in I_{a b}}\left(S_{a i}\right)^{2} \sum_{i \in I_{a b}}\left(S_{b i}\right)^{2}}}
$$

where $S_{a i}$ and $S_{b i}$ are the historical preference of $U_{a}$ and $U_{b}$ on item $i$ respectively.

In order to prevent the huge number of users that may reduce the deviation, we separate our data set into five equal groups, and the average similarity into three classes: (1)Average similarities among users in the independent trust group. (2)Average similarities among other users in the group. (3)Average similarities of (1) and (2). The average similarities among users of each group are shown in Table .1. In the five groups, the similarities among users in class 1 
Table 1: AVERAGE SIMILARITY

\begin{tabular}{|l|l|l|l|}
\hline \multicolumn{1}{|c}{ Table 1: AVERAGE SIMILARITY } \\
\hline Group 1 & 0.5692 & 0.5176 & 0.5351 \\
\hline Group 2 & 0.4856 & 0.4783 & 0.4814 \\
\hline Group 3 & 0.4689 & 0.4529 & 0.4593 \\
\hline Group 4 & 0.4878 & 0.4585 & 0.4662 \\
\hline Group 5 & 0.5351 & 0.4982 & 0.5174 \\
\hline
\end{tabular}

are more than those of class 2 and class 3 , from which we can see that users in the independent group have bigger similarity than other users, which indicates that the independent group does have influence on users' preference.

Table 2: EXPERIMENTAL WEIGHT SEQUENCES

\begin{tabular}{|c|c|c|c|c|c|}
\hline & Sequence 1 & Sequence 2 & Sequence 3 & Sequence 4 & Sequence 5 \\
\hline$\alpha$ & 4 & 6 & 7 & 9 & 12 \\
\hline$\beta$ & 0.5 & 0.7 & 0.8 & 0.4 & 0.7 \\
\hline
\end{tabular}

Experiment 2. Impact of parameters $\alpha$ and $\beta$.

$\alpha$ and $\beta$ are corrected parameters for the influence of the number of vertexes on bipartite graphical correlation in Eq.2. We need to figure out the suitable values for $\alpha$ and $\beta$ to achieve the best prediction accuracy of the data set we used. We examine the influence of parameters $\alpha$ and $\beta$ on the accuracy of prediction based on the MovieLens-t which are the training set selected from MovieLens. In this experiment, the predictions are got from the values of bipartite graphical correlation. We recommend the Top $n$ items which have the largest values of bipartite graphical correlation. To improve the accuracy of the experiment, after repeating experiments and tests, we divide the MovieLens-t into two different data sets, in which one choose $40 \%$ of the data as training set, and another $60 \%$. After repeating experiments, we set $C_{t h d}=4, I d_{t h d}=2$, and choose six sequences of weight coefficients (Table.2) to select the optimal combination. Because MovieLens-t are changing with every operation, we run the algorithm five times on each weight coefficients, and record the average values. The results are shown in Fig.5 and Fig.6, from which we can see that with the increasement of $R$, the five sequences show an increasing trend. When $R=5$, they achieve the best $P @ R$, and the weight sequence 3 can achieve better prediction accuracy than other sequences in both $40 \%$ and $60 \%$ training sets. In other words, sequence 3 , 

$\alpha=7, \beta=0.8$ in the following experiments.

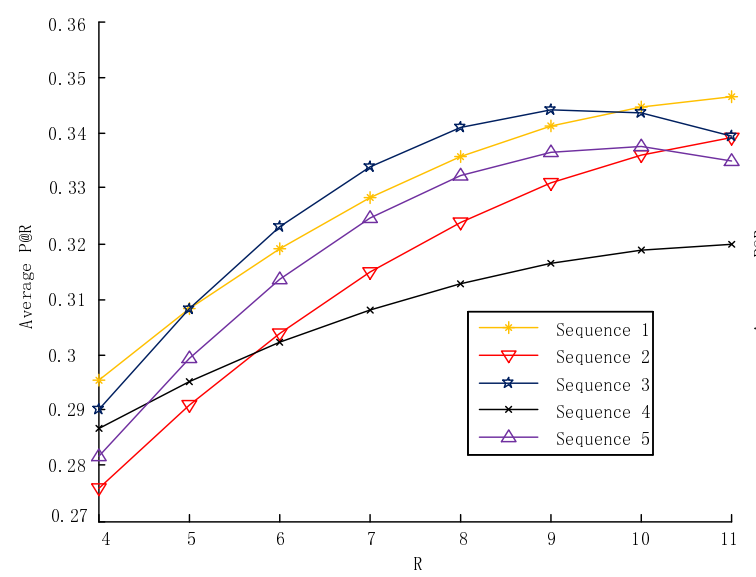

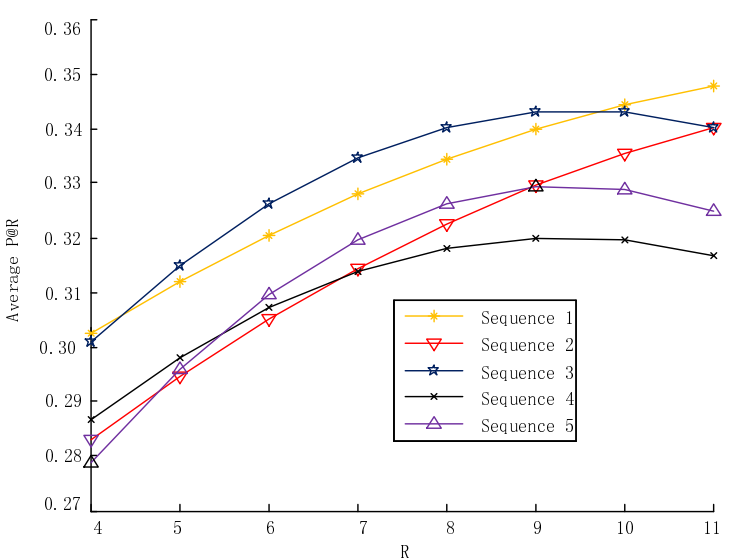

Figure 5: Impact of $\alpha$ and $\beta$ on $P @ R$ (40\% as training Figure 6: Impact of $\alpha$ and $\beta$ on $P @ R(60 \%$ as training set) set)

Experiment 3. Impact of parameters $\chi$

The purpose of this experiment is to obtain the optimal ratio of bipartite graph correlation and implicit trust when obtaining users' preference. The value of $\chi$ is the weight of the two factors in Eq.9, its value space is $[0,1]$. After repeating experiments, we select several representative values of $\chi$ (Table.3) to get the optimal weight. Judging from the experimental results(Fig.7 and Fig.8), we can see that sequence 4 has better performance than other sequences, which indicates that the proposed algorithm can achieve best $P @ R$ when $\chi=0.62$. It can also be found from the results that although the bipartite correlation and implicit trust both have influences on acquiring the preferences, the bipartite correlation reflect users' preference more accurately.

Table 3: EXPERIMENT WEIGHT SEQUENCES

\begin{tabular}{|c|c|c|c|c|c|}
\hline & Sequence 1 & Sequence 2 & Sequence 3 & Sequence 4 & Sequence 5 \\
\hline$\chi$ & 0.24 & 0.32 & 0.46 & 0.62 & 0.83 \\
\hline
\end{tabular}

\section{Experiment 4. Comparison with other methods}

We compare our algorithm with RNCF[16] and GBR[19] in the test data set of MovieLens and conceal the users' data randomly by ABO. The GBR uses directed trust graph to generate recommendation which is one of the latest applications of graph model on recommender systems. Comparing with other existing methods, such as [17] and [18], it is more relevant to our method 


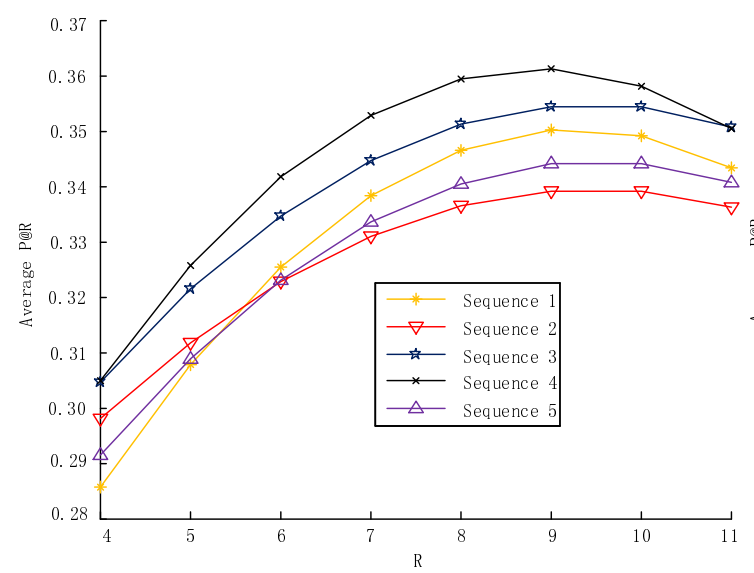

Figure 7: Impact of $\chi$ on $P @ R(40 \%$ as training set)

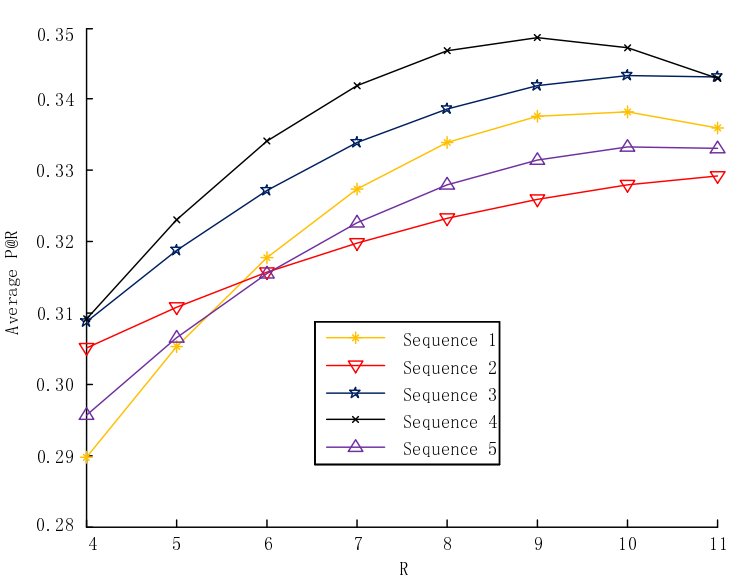

Figure 8: Impact of $\chi$ on $P @ R(60 \%$ as training set $)$

which is based on bipartite graphical correlation and implicit trust, the comparison with which can more objectively reflect the real capability of our method. The RNCF uses rating normalization to improve most of methods known as collaborative filtering based recommender systems. Then we compute the items' predicted ratings by using the three algorithms, and $P @ R$ is employed to measure the deviation. In experiment 2 and 3 , we have validated the optimal values of parameters $\alpha, \beta$ and $\chi$ in different training set scales ( $40 \%$ and $60 \%$ ), and used these optimal values in the comparison with other works. The training set is selected to test the parameters in our method, while the other two methods are tested directly on the rest data set(test set).

In the first experiment, we selected about $40 \%$ of the data randomly as training set, including 260 users' 38000 historical behaviors on 680 movies. Fig.9 reports the average $P @ R$ of our algorithm, GBR and RNCF on different R. It can be found that the $P @ R$ of the three algorithms are increasing with the increasement of $\mathrm{R}$, which means that the accuracy of prediction can be improved by increasing the number of movies in recommendation list. The possible reason is that if we have a bigger recommendation list, there is a higher probability to meet users' preference(but in real applications, its scale should be controlled in suitable range). Another finding is that the $P @ R$ of our method is larger than that of GBR and RNCF in Fig.9. The average $P @ R$ of our method is 0.344 , while those of GBR and RNCF are 0.319 and 0.324 respectively. If we increase the scale of training set to $60 \%$, we can see from Fig.10 almost all the three methods have a better performance. The average $P @ R$ of our method, GBR and RNCF are $0.352,0.323$,and 0.328 respectively. Comparing the Fig.9 with Fig.10, it can be found that the scale of test set has also influenced the accuracy of GBR and RNCF, and our method has achieved a better 
prediction accuracy about $7 \%$ than GBR and RNCF. These results have provided empirical evidence that the use of graph model and independent trust group can lead to an improved predictive performance.
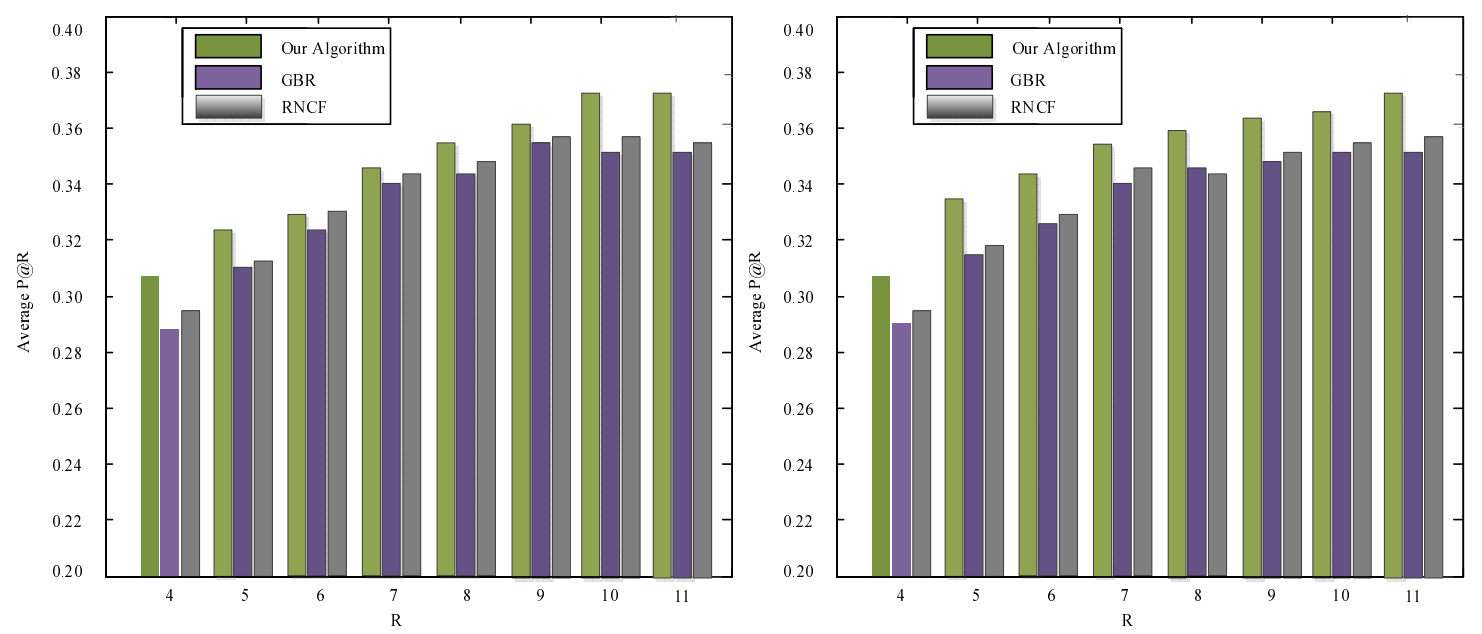

Figure 9: Comparison of our algorithm with $G B R$ and Figure 10: Comparison of our algorithm with $G B R$ and $R N C F(40 \%$ as training set) $R N C F(60 \%$ as training set)

\section{Conclusion and discussion}

In this paper, we focus on the study of recommender approaches to address the problems suffered by most of the existing approaches, such as the influence of the sparse data set on their prediction accuracy. To solve these problems, a preference elicitation method is developed by using the bipartite graph correlation and implicit trust. Specifically, at the beginning, this method gets the correlation values between users and items by using Markov separate algorithm. Then it structures the independent trust group and abstracts the correlation among users as explicit/implicit trust to compute the implicit trust according to the non-binary trust graph. Finally, a weighted prediction algorithm based on bipartite graph correlation and implicit trust is employed to compute the final ratings.

Experiments in very sparse data set have demonstrated that our method can achieve better $P @ R$ comparing with existing methods. This indicates that in this paper, we have found a new way to generate prediction by using graph model and trust relationship. It is proved that the bipartite graphical correlation and independent trust group can improve the accuracy of prediction. Compared with the existing works, the bipartite graphical correlation which is raised 

influence factors and measure method.

Besides, compared with the existing methods, our method has a better performance and a different increasing trend under most circumstances. The difference may relate to the data itself and the values of parameters. The possible reason is that there are exists lots of irrelevant or useless preference records, thus if we choose these records to get prediction, it may lead to a dissatisfied result. Furthermore, although the optimal values of parameters in various experimental data are different, in order to ensure the objectivity, we have to choose the eclectic option in one experiment.

In further research, the following points need to be addressed: (1) How to overcome the influence of different data set on the prediction accuracy by using graph model?(2)How to improve the diversity and accuracy at the same time? (3) How to combine the context information with graph model?

\section{Acknowledgment}

The authors want to appreciate all the reviewers for their insightful comments and suggestions which help to make this paper more readable. This work is partly supported by NSAS research program of NorthWest University in China, and its also supported by the National Science Foundation of China under Grant No.61373146, Ph.D Programs Foundation of Ministry for Education of China under Grant No.20116101110016. ShaanXi Provincial Natural Science Foundation of China under Grant No.2014K05-42, Foundation of ShaanXi Educational Committee under Grant No.11JK1059, Foundation of ShaanXi Science Innovation under Grant No.2012ZKC05-2, Foundation of 2013 Science Research of ShaanXi Educational Committee under Grant No.2013JK1178.

\section{References}

[1] H.Alharthi, D.Inkpen, Content-based recommender system enriched with wordnet synsets, in: Conference on intelligent text processing and computational linguistics, Springer Lecture Notes in Computer Science, Cairo, Egypt, 2015, pp. 295-308.

[2] J. Wintrode, G. Sell, A.Jansen, Content-based recommender systems for spoken documents, in: IEEE international conference on acoustics, speech, and signal processing, IEEE, Brisbane,Australia, 2015, pp. 5201-5205. 
[10] X.Luo, M.Zhou, Y.Xia, An efficient non-negative matrix-factorization-based approach to collaborative filtering for recommender systems, IEEE transactions on industrial informatics 10 (2) (2014) 1273-1284.

[11] H.Kim, I.Ji, I.Ha, G.Jo, Collaborative filtering based on collaborative tagging for enhancing

[12] F. G. C.Leung, S.Chan, A probabilistic rating inference framework for mining user preferences from reviews, World wide web internet web information systems 14 (2) (2011) 187-215.

[13] S.Wei, N.Ye, S.Zhang, X.Huang, J.Zhu, Collaborative filtering recommendation algorithm

[3] J.Bobadilla, F.B.Ortega, A.Hernando, A.Gutierrez, Recommender systems survey, Knowledge based systems 46 (1) (2013) 109-132.

[4] H.Liu, Z.Hu, A.Mian, A new user similarity model to improve the accuracy of collaborative filtering, Knowledge based systems 56 (3) (2014) 156-166.

[5] J. Y.Xu, Collaborative recommendation with user generated content, Engineering applications of artificial intelligence 45 (2) (2015) 281-294.

[6] K. Chung, Effect of facial makeup style recommendation on visual sensibility, Multimedia tools and applications 72 (2) (2014) 843-853.

[7] I.Cantador, An enhanced semantic layer for hybrid recommender systems, Semantic web: ontology and knowledge base enabled tools, services, and applications 7 (1) (2011) 44-78.

[8] E.Wiranto, S.Hartati, R.Wardoyo, Improving the prediction accuracy of multicriteria collaborative filtering by combination algorithms, International journal of advanced computer science and applications 5 (4) (2014) 51-62.

[9] D. Anand, K.K.Bharadwaj, Exploring graph-based global similarity estimates for quality recommendations, International journal of computational science and engineering 9 (3) (2014) 188-197.

the quality of recommendation, Electron commerce Research and Applications 9 (1) (2010) 73-83.

based on item clustering and global similarity, in: 2012 Fifth international conference on business intelligence and financial engineering, 2012, pp. 60-72. 
[14] B.Wang, C.Wang, J.Bu, C.Chen, W.V.Zhang, Whom to mention: expand the diffusion of tweets by recommendation on micro-blogging systems, in: Proceedings of the 22nd international conference on World Wide Web, 2013, pp. 1331-1340.

[15] W.Zhao, Z.Y.Guan, Z.Liu, Ranking on heterogeneous manifolds for tag recommendation in social tagging services, Neurocomputing 148 (4) (2015) 521-534.

[16] S.C.Kim, K.J.Sung, C.S.Park, Improvement of collaborative filtering using rating normalization, Multimedia tools and applications 62 (2) (2013) 1-12.

[17] J. Z.X.Liu, N.Zhang, Personalized recommendation algorithm based on collaborative filtering and network structure, Complex System and Complexity Science 8 (2) (2011) 168-179.

[18] K. Lee, Escaping your comfort zone: A graph-based recommender system for finding novel recommendations among relevant items, Expert systems with applications 10 (42) (2015) $4851-4858$.

[19] H.D.Zhong, S.Zhang, Y.Wang, Y.Shu, Study on directed trust graph based recommendation for e-commerce system, International journal of computers communications and control 9 (4) (2014) 510-523.

[20] X.N.Lam, T.Vu, T.D.Le, Addressing cold-start problem in recommendation systems, in: Proceeding of the 27th annual international ACM SIGIR conference on research and development in information retrieval, ACM, NewYork,USA, 2008, pp. 208-211.

[21] L.Becchetti, UM.Colesanti, A.Marchetti-Spaccamela, A.Vitaletti, Recommending items in pervasive scenarios: models and experimental analysis, Knowledge information system 28 (3) (2011) 555-578.

[22] H.Wang, W.J.Li, Relational collaborative topic regression for recommender systems, IEEE Transactions on Knowledge and Data Engineering 27 (5) (2015) 1343-1355.

[23] B.K.Patra, R.Launonen, V.Ollikainen, A new similarity measure using bhattacharyya coefficient for collaborative filtering in sparse data, Knowledge based systems 82 (2) (2015) 163-177.

[24] D. P.Bocchini, S.Duygu, Efficient accurate and simple markov chain model for the lifecycleanalysis of bridge groups, Structural safety 40 (2013) 178-186. 